\title{
The effect of particle shape on the marginal rigidity state in 2D granular media
}

\author{
L. Sibille ${ }^{1(a)}$, T. Mullin ${ }^{2}$ and P. Poullain ${ }^{1}$ \\ ${ }^{1}$ Laboratory GeM, Université de Nantes, CNRS - BP 420, 44606 Saint-Nazaire Cedex, France, EU \\ ${ }^{2}$ Manchester Centre for Nonlinear Dynamics, University of Manchester - Oxford Road, \\ Manchester, M13 9PL, UK, EU
}

PACS 45.70.-n - Granular systems

PACS 45.50.-j - Dynamics and kinematics of a particle and a system of particles

\begin{abstract}
Experimental results from an investigation of the assembly of two-dimensional granular piles are presented. Three different particle shapes were used in the investigation of the evolution of piles towards isostatic and isotropic granular assemblies. Good agreement is found with previous experimental results for all particle shapes. We attempt to classify the divergence of a characteristic length-scale of the system as a power law and provide an estimate of the critical exponent. We find that the results are independent of particle shapes and speculate this may be a general feature of two-dimensional convex particles.
\end{abstract}

Introduction. - Interesting and often counterintuitive properties of collections of granular materials have attracted the attention of physicists, where the tools of condensed matter physics have been brought to bear [1-3]. Dense assemblies of granular materials can exhibit both solid and liquid-like behavior. Moreover, materials such as soils can switch state from solid to liquid-like under cyclic [4] or even constant loading [5]. This transition between behavior types constitutes an important problem in civil engineering.

It is argued [6] that the static equilibrium of a twodimensional assembly of $N_{g}$ frictional and rigid particles requires at least $3 N_{g}$ equations for the forces and torque to balance. When friction is taken into account, each particle contact involves both normal and tangential components of the force. Thus, static equilibrium of an assembly of $N_{g}$ particles with $N_{c}$ contacts is only assured when $2 N_{c} \geqslant 3 N_{g}$, which corresponds to a minimum contact number $N_{c} \geqslant 3 N_{g} / 2$ and a minimum coordination number (mean number of contacts per particle) $z \geqslant z_{c}=3$ in two dimensions (see also [7]). When $z=z_{c}$, the granular assembly corresponds to an isostatic mechanical system and results from discrete numerical simulations and experiments $[6,8-10]$, corroborate that $z_{c}=3$ constitutes a lower boundary for "solid" bi-dimensional granular assemblies in quasi-static equilibrium. The evolution of

\footnotetext{
(a) E-mail: luc.sibille@univ-nantes.fr
}

a granular assembly from a "solid" to a "liquid" state is expected to be characterized by a coordination number equal to $z_{c}$ [11], although, this has not been demonstrated conclusively in an experiment.

The marginal rigidity occurs when $z=z_{c}$ [12-14]. Since a granular assembly at this condition constitutes an isostatic system, it is believed that the distribution of stress transmission can be obtained from the geometry of the contact network $[7,14,15]$. In order to test these ideas experimentally the marginal rigidity state was accessed by building granular piles on a horizontal plane in two dimensions using a belt feed system $[12,13]$. This is argued to be analogous to free fall without the dynamic factor of impact resulting from gravity. It is shown that for increasing values of the initial density of the particles, the coordination number in the final pile decreases until a value close to $z_{c}=3$ is achieved i.e. it is equivalent to the marginal rigidity state discussed above. During the construction of the pile a yield front of rearranging particles is formed between the oncoming particles and the consolidated pile where the size of the front can be considered as the number of particles within the region. The depth of the front in terms of normalized density is expected to diverge as a power law when the marginal rigidity state is approached, suggesting a critical phenomenon.

Available evidence suggests that the exponent of the power law is close to one, but it may be argued the size 


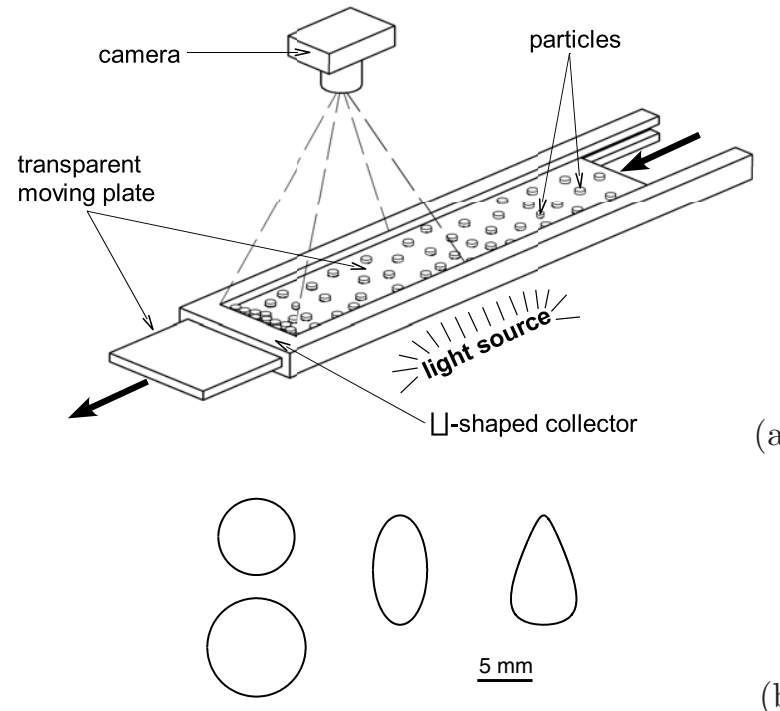

(a)

(b)

Fig. 1: (a) Sketch of the experimental device, particles are placed on a plate moving toward a $\sqcup$-shaped collector; (b) twodimensional particles: bidisperse discs, ellipse and with a pear shape (relative sizes have been respected in the figure).

of the system investigated was too small to give complete confidence in this value [13]. The experiments were performed with particles with an elliptical cross-section and it is suggested that the critical exponent ought to be insensitive to details of the particle shape so that the findings might be applicable to a wide range of frictional bi-dimensional granular materials. Here we report the results of an experimental investigation into the construction of granular piles with three different particle shapes: discs, ellipses, and pear shapes. Images of the particles captured during the construction of the pile were used to characterize both the consolidated pile and the yield front. The effect of particle shape on the marginal rigidity state and the front size divergence is discussed, and an estimate of the critical exponent is given.

Experimental details. - The piles were approximately two-dimensional and were assembled in the manner suggested by Blumenfeld et al. [13]. A schematic diagram of experimental device is presented in fig. 1a. Particles were placed on a $1 \mathrm{~cm}$ thick Perspex plate which was translated at constant speed towards a $\sqcup$-shaped collector which was $160 \mathrm{~mm}$ wide. Hence, particles were carried along by the moving plate until they encountered either the wall of the collector or other particles in the growing pile. Once the free translation of particles was stopped, further movement took place as the particles slid on the moving plate and an analogue of gravitational force was produced by frictional interaction between particles and the plate. A high resolution camera with $4288 \times 2848$ pixels i.e. 16 pixels $/ \mathrm{mm}$, was fixed above the experiment which was illuminated from below and pictures of the
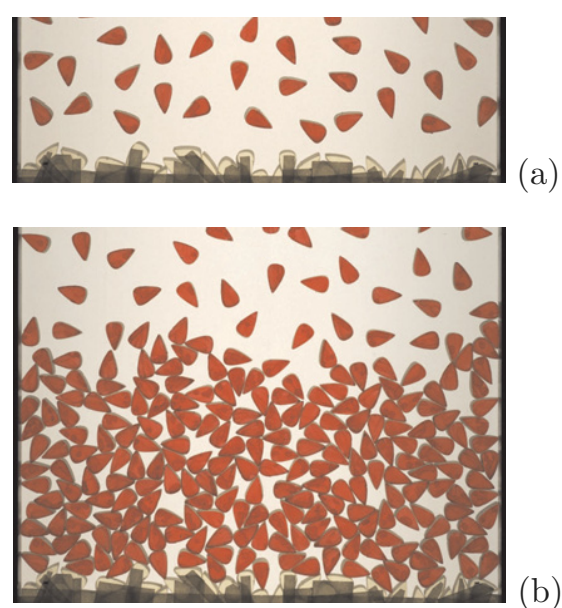

Fig. 2: (Colour on-line) Construction of a granular pile with pear-shaped particles, (a) at the start of the test, (b) near the end of the test.

growing pile were captured at predetermined regular time steps. All the experiments were performed under quasistatic conditions since a low velocity, $v_{i}=25 \mathrm{~mm} / \mathrm{min}$, was chosen for the moving plate.

The particles were all cylinders of height $6.6 \pm 0.1 \mathrm{~mm}$ with the three different cross-sections shown in fig. $1 \mathrm{~b}$ (see also [16]). The first set of particles consisted of a binary mixture of 144 disks of $6.9 \mathrm{~mm}$ diameter and 144 disks with diameter $8.9 \mathrm{~mm}$. The mixture was chosen to minimize the possibility of the formation of regular fabrics in the granular piles. The second set of particles consisted of 280 ellipses with dimensions $9.9 \mathrm{~mm}$ and $4.9 \mathrm{~mm}$ on the major and minor axes, respectively. The third set comprised 280 pear-shaped particles which were $9.9 \mathrm{~mm}$ long and $4.9 \mathrm{~mm}$ wide as shown in fig. 1b. The initial conditions for the experiments were set by placing particles by hand at random positions on the plate with the constraint of no contacts. A more sophisticated procedure was initially tried involving creating a pattern of locations using a randomization algorithm. This proved to be impractical and placement by eye was found to be satisfactory and the following checks were made. The density $\rho_{i}$ was verified to be homogeneous by dividing the initial granular assemblies into several virtual layers, and ascertaining that the local density did not differ by more than $\pm 7 \%$ from the density of the whole assembly. The assembly was also checked to be isotropic with respect to particle orientations for both elliptical and pear-shaped grains.

The ratio $\eta$ between the width of the collector and the mean particle size $\phi$ (for each particle shape) was $\eta=20.3$ for the discs and $\eta=21.6$ for the ellipses and the pears. This value was a compromise between minimizing the effects of the sidewalls and the computational time required for image analysis. As shown in fig. 2, the bottom of the $\sqcup$-shaped collector had appropriate particles glued to it in order to minimize the influence of the otherwise flat end-wall of the collector on the fabric of the granular pile. 
Experiments were performed for each particle shape, by building piles with different initial densities $\rho_{i}$. For each initial density and particle shape, tests were repeated ten times and a total of 260 tests were realized. Each experimental run produced a set of snapshot images of the processes involved in pile formation. Information concerning position and orientation of the particles as well as the number of contacts was extracted from each of the images.

Two different image processing procedures were used, depending on the information required. In order to determine the positions and orientations of particles in each image, image segmentation based on the selection of sets of pixels with respect to their colour was performed where each particle was ascribed a given set of pixels. Grain position was determined from the centre of mass of the pixel set. The second moment of inertia of the pixel set about a prescribed direction $\Omega$ was calculated. The particle orientation for ellipses and pears was computed as the angle between the direction $\Omega$ and the major axis of an ellipse or a pear resulting in the same second moment of inertia of the pixel set. The direction $\Omega$ was chosen to be parallel to the translation direction of the moving plate.

The computation of the number of contacts from an image required a more sophisticated procedure. Once the centre of mass and the orientation of each particle had been established using the method described above, the number of contacts could, in principle, be estimated by representing each particle by an idealized model of a disc, ellipse or pear. However, the actual geometry of each particle differed slightly from the idealized model. Consequently, a tolerance was introduced to obtain estimates of the coordination number $[8,9]$. In order to reduce errors incurred using idealized models of particles, the computation of the number of contacts was instead based on the detection and recognition of particle edges. The edge of each particle was detected in images by performing segmentations with the "GVF snake" developed by $\mathrm{Xu}$ and Prince [17] and based on the method of active contours. We define the spacing $s$ as the distance between the apparent (or detected) edges of two neighbouring particles. The apparent spacing $s$ was measured with an accuracy of 1 pixel (i.e. $0.0625 \mathrm{~mm}$ ). However, errors in edge detection, and small tilts of some particles sometimes produced an apparent non-vanishing spacing $s$ between two particles which were actually in contact. The apparent coordination number computed for apparent contacts with a spacing $s$ lower than a given threshold $s^{*}$ is plotted in fig. 3. The true coordination number $z$ for a vanishing spacing was determined from the extrapolation of a linear approximation [13].

Characterization of the granular pile. - Three phases were distinguished during the construction of all of the granular piles (see fig. 6): i) the particles were not in contact and were simply carried along by the moving plate, ii) the consolidated pile was composed of particles which

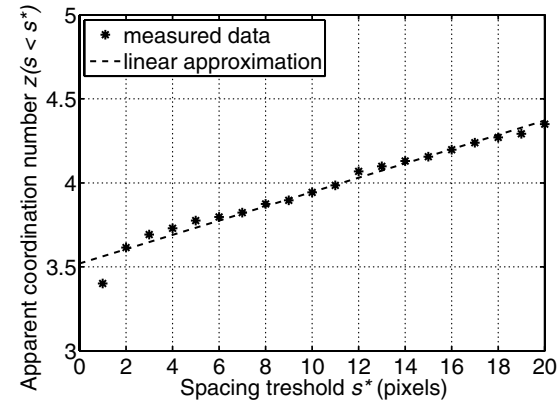

Fig. 3: Apparent coordination number computed for apparent contacts with a spacing $s<s^{*}$ (for a pile built with ellipses).

had achieved a steady state $^{1}$, iii) a yield front between these two phases including particles in a consolidation process, i.e. they had not reached a steady state.

The consolidated pile was characterized in terms of its density $\rho$, coordination number $z$ and fabric anisotropy $a$. We define the density $\rho$, on a surface of area $S$ including particles occupying an area $S_{g}$, as $\rho=S_{g} / S$. Zones within a mean particle width of the sidewalls, characterized by a lower density than the mean value taken over the whole consolidated pile, were not taken into account in the computation of the pile density. The evolution of the consolidated pile density $\rho_{p}$ is shown in fig. 4 a plotted as a function of the initial density $\rho_{i}$ where each point was estimated from ten repetitions of the experiment. The variations in $\rho_{p}$ are small for all the particle shapes but the trend is statistically significant. For the discs, $\rho_{p}$ is slightly lower than the density of a square arrangement of mono-disperse discs, $\rho=\pi / 4=0.785$, and close to the density of a random loose packing of hard discs $(\rho=0.772)$ [18]. for ellipses and pears, rotation of particles is partially locked because of the shape anisotropy. Consequently, rearrangement of the particles is restrained and $\rho_{p}$ is lower than for disks.

The coordination number $z$ was computed on the same part of the consolidated pile as the pile density (zones close to the walls were excluded). Values of $z$ with respect to $\rho_{i}$ are shown in fig. 4b. For discs, at low initial density, the coordination number approaches 3.8 which is in good agreement with the highest values of $z=4$ found from discrete numerical simulations [6]. For all particle shapes, after a slight decrease in $z$ with increasing $\rho_{i}, z$ is seen to stabilize at values of $\approx 3.4$ (disks and ellipses), and $\approx 3.2$ (pears).

The fabric of the granular piles can be characterized by the vector branch joining the centres of mass of particles in contact. A histogram of $N(\theta) / N_{c}$ where $N(\theta)$ is the number of vector branches belonging to a range of mean orientation $\theta$, and $N_{c}$ is the total number of contacts is shown in fig. 5. A least squares fit to the variation in the

${ }^{1}$ The notion of steady state is relative, since while the pile was growing, the stress at the bottom of the pile evolved slowly and could lead to some modification of the structure of the pile. 


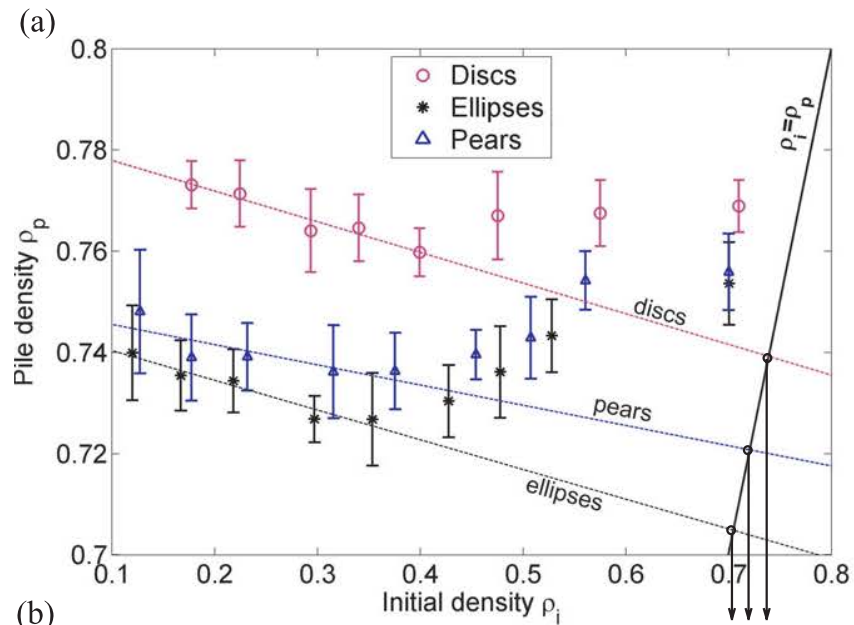

(b)

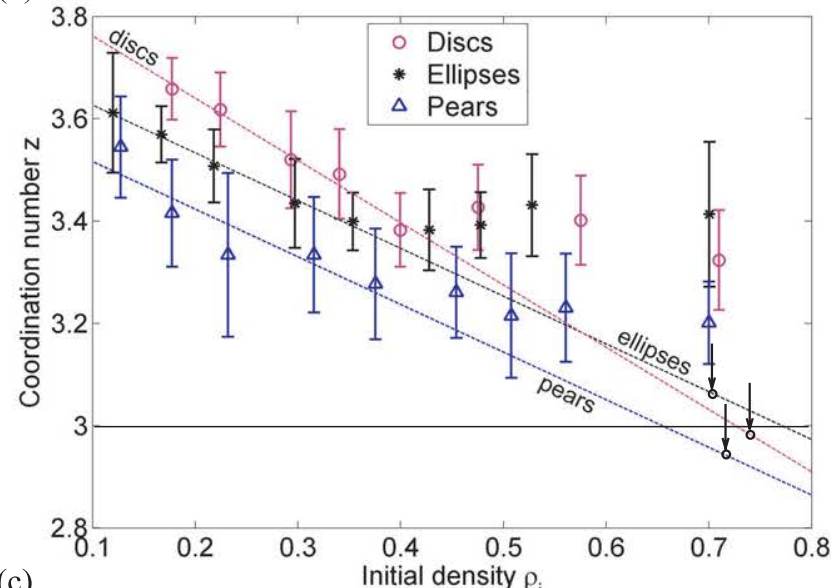

(c)

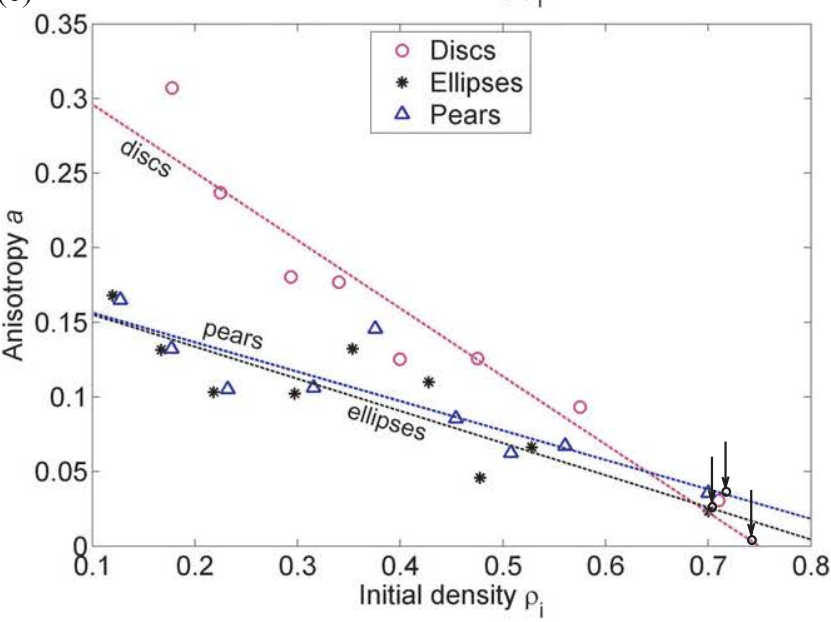

Fig. 4: (Colour on-line) Pile density (a) and coordination number (b) vs. the density $\rho_{i}$ of the falling particles; error bars represent the standard deviation; dashed lines represent linear approximations for the smallest five values of $\rho_{i}$. (c) The fabric anisotropy is determined from the distribution of the orientations of the branch vectors (see fig. 5).

data is given by the function

$$
N(\theta)=m\left[1+a \cos \left(4 \theta+\theta_{0}\right)\right],
$$

where $a$ represents the fabric anisotropy. The variation of $a$ with increasing $\rho_{i}$ is presented in fig. $4 \mathrm{c}$. It can be seen that

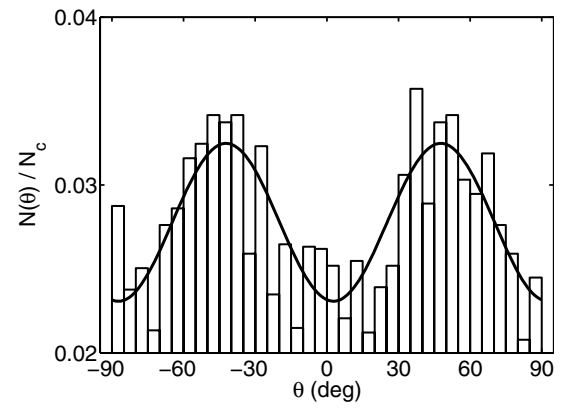

Fig. 5: Histogram of the branch vector orientations for the ellipses and $\rho_{i}=0.12$, the continuous line represents the fit defined in eq. (1).

$a$ decreases monotonously when $\rho_{i}$ increases for all particle shapes. For high $\rho_{i}$ values, the fabric of the pile tends to be isotropic. The pears and ellipses had identical ratios between the major and minor axes, i.e. similar particle shape anisotropies, and the observed values of $a$ were very close to each other as might be expected.

The variations in $\rho_{p}$ and $z$ can be analyzed in the manner suggested by Blumenfeld et al. [13]. Initially we consider, for each particle shape, the points corresponding to the smallest five $\rho_{i}$ values in figs. $4 \mathrm{a}$ and $\mathrm{b}$. The remaining data points will be discussed later. For the first sets of five points, the pile density $\rho_{p}$ and the coordination number $z$ decreases with increasing initial density $\rho_{i}$. This evolution is well described by a linear least squares fit approximation as shown in figs. $4 \mathrm{a}$ and b. By extrapolating these approximations to the values for $\rho_{p}$, we find a limit density $\rho_{c}$ for which $\rho_{p}=\rho_{i}$ with $\rho_{c}^{\text {Discs }}=0.739$, $\rho_{c}^{\text {Ellipses }}=0.705$ and $\rho_{c}^{\text {Pears }}=0.721$. Granular piles at these limit densities $\rho_{c}$ can also be characterized by extrapolated coordination numbers $z_{c}$ with $z_{c}^{\text {Discs }}=2.98$, $z_{c}^{\text {Ellipses }}=3.06$ and $z_{c}^{\text {Pears }}=2.94$. These values provide a good match with the analytical results for an isostatic granular assembly of frictional particles $z_{c}=3$, with a relative error $\leqslant 2 \%$.

The experimental results indicate that the coordination number $z$ does not monotonically decrease until it reaches $z_{c}$ with increasing $\rho_{i}$, but, instead, $z$ appears to stabilize at a fixed value $(\approx 3.4$ for disks and ellipses and $\approx 3.2$ for pears) as discussed above. As the granular pile tends toward an isostatic system with $z_{c}=3$, the stability of the pile provided by the contact network becomes less and less assured. In other words, the contact network of an isostatic system readily evolves, after a "small disturbance", into an hyperstatic and more stable state. It is, perhaps, a necessary consequence of "imperfections" which will be present in any experiment, that granular piles below a given $z$ value are not sufficiently stable to reach the theoretical limit of $z_{c}=3$. Nevertheless, fabrics of granular piles evolve, as shown in fig. 4c where it can be seen that the anisotropy continues to decrease even after $z$ saturates. Extrapolations of the linear approximations 


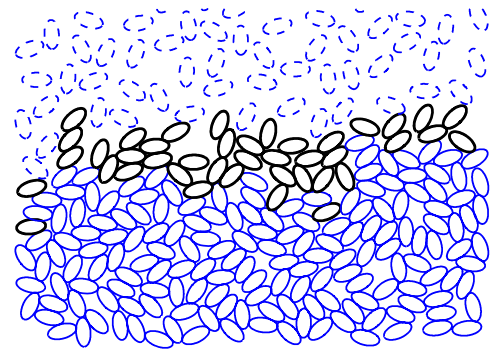

Fig. 6: (Colour on-line) Ellipses with thick edges form the yield front; ellipses in dashed line are in analogue free fall, the consolidated pile is composed of ellipses with thin continuous edges $\left(\rho_{i}=0.30\right)$.

indicate that the fabric approaches an isotropic state when $\rho_{i}=\rho_{c}\left(a_{c}^{\text {Discs }} \approx 0, a_{c}^{\text {Ellipses }}=0.025\right.$ and $\left.a_{c}^{\text {Pears }}=0.034\right)$. The increase of $\rho_{p}$ when $z$ stabilizes is still an open question, but constitutes experimental evidence that $\rho_{p}$ depends on both $z$ and fabric anisotropy $a[6,10]$.

Characterization of the yield front. - We consider now the yield front formed at the top of the granular pile including particles involved in the consolidation process. Such particles are defined at a given time as particles in direct contact with others either in the pile or in the front itself but having not yet reached a final position [13]. In practice, a particle was attributed to the yield front if its translation displacement between two sequential images was higher than $2 \%$ of the displacement of the moving plate (fig. 6). The identification of the yield front was performed in the same way for all particle shapes and any rotations of the particles was ignored.

We denote the velocity of the particles in free motion by $v_{i}$, and that of the centre of mass of the front by $v_{f}$. A local frame of reference with respect to the front can be defined such that particles enter in the front at a velocity $v_{r}=v_{f}+v_{i}$ with a density $\rho_{i}$. They leave the front at $v_{f}$ with a density $\rho_{p}$, since they are now within the consolidated pile. From mass conservation, the following relationships between velocities can be established:

$$
v_{f}=v_{i} \frac{\rho_{i}}{\rho_{p}-\rho_{i}},
$$

and $v_{r}=v_{f}+v_{i}=v_{i} \rho_{p} /\left(\rho_{p}-\rho_{i}\right)$. In order to estimate the evolution of the depth of the front $\xi$ with increasing $\rho_{i}$ we consider the time $\tau$ taken by a particle to pass through the front and to reorganize [13]. If we assume that the particle has a constant velocity $v_{r}$ (this is a good approximation when $\rho_{i} \rightarrow \rho_{p}$ ) during the transit of the front, the transit time is given by

$$
\tau \approx \xi / v_{r}=\Delta \rho \xi / v_{i} \quad \text { with } \quad \Delta \rho=\left(\rho_{p}-\rho_{i}\right) / \rho_{p} .
$$

An upper limit of the lateral translation $d_{l}$ of a particle during the crossing of the front is of the order of

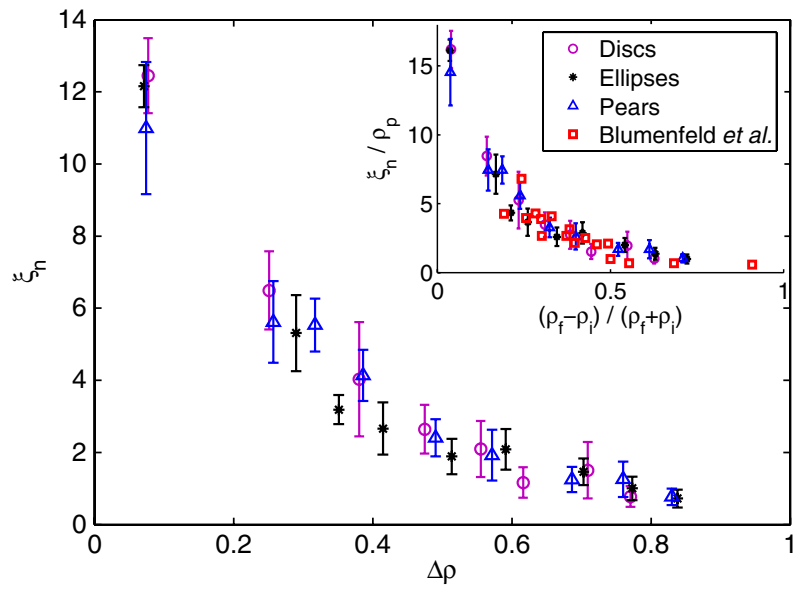

Fig. 7: (Colour on-line) Evolution of dimensionless front depth $\xi_{n}$ with respect to $\Delta \rho$, the inset figure shows a comparison of the same results with those published by Blumenfeld et al. [13] for ellipses (the definition of dimensionless variables by these last authors is slightly different).

$d_{l} \approx \tau v_{i}[13]$. By combining with relation (3), the front depth $\xi$ can be expressed as

$$
\xi \approx d_{l} / \Delta \rho .
$$

$\xi$ and $d_{l}$ can be normalized, for each particle shape, with respect to the mean particle size $\phi$. Let us denote by $N_{f}$ the number of particles involved in the front, then we can define the dimensionless front depth by $\xi_{n}=\xi / \phi=N_{f} / \eta$ and $\xi_{n} \approx d_{l} /(\phi \Delta \rho)$.

The evolution of the dimensionless front depth $\xi_{n}$ vs. $\Delta \rho$ is shown in fig. 7. It can be seen by inspection that particle shape does not have a significant influence on the results. A comparison with a depth front measured by Blumenfeld et al. [13] for cardboard ellipses is presented in the inset figure of fig. 7 . The results from different experiments are all in very good agreement with each other showing the robustness of this feature.

As conjectured in [13], it appears from measurements of the lateral translation $d_{l}$ during the transit of the front, that $d_{l}$ converges very slowly towards 0 when $\Delta \rho \rightarrow 0$. Thus we can expect the front depth diverging with a power law as [13]

$$
\xi \propto \Delta \rho^{-\alpha} .
$$

The dimensionless front depth $\xi_{n}$ vs. $\Delta \rho$ is shown plotted on a logarithmic scale for each particle shape in fig. 8 . Points corresponding to the lowest value of $\Delta \rho$ for each particle shape respectively deviate from a power law. These points are clearly influenced by the finite size of our experiment. In this case, almost all of the particles were involved in the front and the front depth was still increasing when experiment was stopped, i.e. a steady front depth has not been reached. However, these three points are interesting since they constitute lower limits of $\xi_{n}$ for low $\Delta \rho$ values. However, they were not taken into account in the estimation of the exponent $\alpha$ of the 


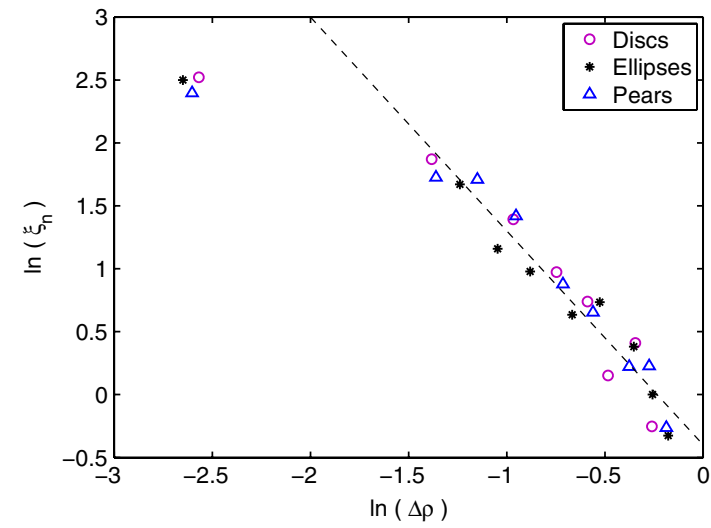

Fig. 8: (Colour on-line) Plot on logarithmic scales of $\xi_{n} v s . \Delta \rho$, the dashed line represents a critical divergence $\xi_{n} \propto \Delta \rho^{-1.7}$.

power law, eq. (5). We find that front depth divergence is accurately described by $\alpha=1.7$, for all particle shapes.

Discussion and conclusion. - We have shown that characteristics of the final consolidated pile in terms of density $\rho_{p}$, coordination number $z$ and fabric anisotropy $a$ are all a result of particle reorganization in the yield front. At very low initial densities $\rho_{i}$, the front is less than one particle layer thick. The particles are not in direct contact with each other and hence do not interact. Therefore, each incoming particle to the pile is "free" to find a stable position and forms at least two contacts with particles involved in the consolidated pile. The subsequent compressive force, which we argue is an analogue of gravity, leads to highly anisotropic granular piles.

For larger initial densities, the front size increases, and it can comprise one or more layers of particles. In the front, particles interact with their neighbors restricting the possibilities of translational and rotational motion. In this case, the fabric of a granular pile is not uniquely related to the analogue gravitational force but also depends on the increased number of inter-particle interactions in the front. Since particle reorganizations are restricted in the dense limit, the fabric of the pile approaches that of the incoming particles, fewer contacts are created and the coordination number decreases. However, the marginal rigidity state is not achieved experimentally, whatever the particle shapes. When approaching an isostatic system where $z=z_{c}$, we expect the pile to contain clusters of particles with $z>z_{c}$ and others with $z<z_{c}$. Consequently, some slight reorganizations will always take place in the pile (preferentially in clusters where $z<z_{c}$ ) preventing the coordination number decreasing below a given value. Density is function of $z$ and $a[6,10]$, and it appears that $\rho_{p}$ increases for $z$ constant with $a$ decreasing.

Despite the above practical limitations, extrapolation of the results indicates convergence towards a marginal rigidity state characterized by $z_{c}=3 \pm 2 \%$ in accordance with that predicted from simple considerations on isostaticity of a contact network. The lack of anisotropy together with the isostaticity of the pile fabric for a unidirectional building process may indicate the limiting state between a liquid-like and solid-like behavior.

The construction of the pile can be characterized by a dimensionless length-scale corresponding to the depth of the yield front [13] which is independent of the particle shape. Moreover, the measured front depth is in good agreement with previous results [13] for cardboard ellipses. When $\Delta \rho \rightarrow 0$, i.e. when approaching the marginal rigidity state, the dimensionless front depth diverges to infinity according to the power law $\xi_{n}=\Delta \rho^{-\alpha}$. This suggests that the marginal rigidity state is equivalent to a critical point. The critical exponent appears insensitive to the particle shape and the rough estimate of $\alpha \approx 1.7$ may be considered as a general property for two-dimensional and frictional particles under quasi-static conditions.

The authors gratefully acknowledge helpful discussions with R. BLUMENFELD and also thank S. LUDING for interesting comments on anisotropy.

\section{REFERENCES}

[1] Jaeger H., Nagel S. and R.P. B., Rev. Mod. Phys., 60 (1996) 1259

[2] De Gennes P., Rev. Mod. Phys., 71 (1999) 5374.

[3] Duran J., Sands Powders and Grains: An Introduction to the Physics of Granular Materials (Springer) 1999.

[4] Seed I., J. Geotech. Eng. Div., 105 (1979) 201.

[5] Darve F., Sibille L., Daouadji A. and Nicot F., $C$. R. Mécanique, 335 (2007) 496.

[6] Rothenburg L. and Kruyt N., Int. J. Solids Struct., 41 (2004) 5763.

[7] Edwards S. and Grinev D., Phys. Rev. Lett., 82 (1999) 5397.

[8] Sibille L. and Froito F., Granular Matter, 9 (2007) 183.

[9] Kolb E., Goldenberg C., Inagaki S. and Clément E., J. Stat. Mech. (2006) P07017.

[10] Rajä̈ F., Troadec H. and Roux S., Key features of granular plasticity, in Granular Materials: Fundamentals and Applications, edited by Anthony S. J., Hoyle W. and Ding Y. (Royal Society of Chemistry, Cambridge) 2004, pp. 157-184.

[11] NG T. and Dobry R., J. Geotech. Eng., 120 (1994) 388.

[12] Ball R. and Blumenfeld R., Philos. Trans. R. Soc. London, Ser. A, 360 (2003) 731.

[13] Blumenfeld R., Edwards S. and Ball R., J. Phys.: Condens. Matter, 17 (2005) S2481.

[14] Ball R. and Blumenfeld R., Phys. Rev. Lett., 88 (2002) 115505.

[15] Blumenfeld R., New J. Phys., 9 (2007) 160.

[16] Zuriguel I. and Mullin T., Phys. Rev. Lett., 98 (2007) 28001.

[17] Xu C. and PRINCE J., IEEE Trans. Image Process., 7 (1998) 359.

[18] Hinrichsen E., Feder J. and Jossang T., Phys. Rev. A, 41 (1990) 4199. 